A method to calculate the surface tension of a cylindrical droplet

This article has been downloaded from IOPscience. Please scroll down to see the full text article.

2010 Eur. J. Phys. 3179

(http://iopscience.iop.org/0143-0807/31/1/008)

View the table of contents for this issue, or go to the journal homepage for more

Download details:

IP Address: 159.226.231.70

The article was downloaded on 29/03/2011 at 09:04

Please note that terms and conditions apply. 


\title{
A method to calculate the surface tension of a cylindrical droplet
}

\author{
Xiaosong Wang and Ruzeng Zhu \\ State Key Laboratory of Nonlinear Mechanics (LNM), Institute of Mechanics, Chinese Academy \\ of Sciences, Beijing 100190, People's Republic of China \\ E-mail: zhurz@lnm.imech.ac.cn
}

Received 10 August 2009, in final form 1 September 2009

Published 4 November 2009

Online at stacks.iop.org/EJP/31/79

\begin{abstract}
The history of Laplace's equations for spherical and cylindrical droplets and the concept of dividing surface in Gibbs' thermodynamic theory of capillary phenomena are briefly reviewed. The existing theories of surface tensions of cylindrical droplets are briefly reviewed too. For cylindrical droplets, a new method to calculate the radius and the surface tension of the surface of tension is given. This method is suitable to be used by molecular dynamics simulations.

(Some figures in this article are in colour only in the electronic version)
\end{abstract}

\section{Introduction}

Capillary phenomena have puzzled human beings since ancient Greece [1]. The rise of water in thin capillaries seems to contradict Newton's law of gravity. Isaac Newton himself mentioned capillary phenomena in his book Opticks [1, 2]. Newton's assistant F Hauksbee observed that the capillary rise of water depends only on the internal diameter of the tube [3]. Therefore, the rise of water in thin tubes could not be interpreted by the gravitational interactions between water and the tube.

Although the concept of the atom had already emerged in ancient Greece, the existence of atoms was not demonstrated experimentally until 1907 by Rutherford and H W Geiger $[4,5]$. The success of Newtonian mechanics led to many attempts to interpret capillary phenomena in terms of interactions between particles. Long before the concept of molecule or particle had been demonstrated experimentally, many scientists who investigated the theories of gases and liquids had widely accepted a speculation that the structure of matter is discrete. Pierre Simon Laplace and others believed that there exists a strong but short-range attractive force between molecules. Based on a concept of internal pressure, Laplace [6, 7] derived a relationship between the surface tension, the pressures of the liquid phase and the vapour phase 
of a spherical droplet. This relationship was derived independently by Thomas Young [3]. Lord Kelvin also obtained this equation in 1858-1859 [8, 9]. This equation can be written as

$$
p_{\alpha}-p_{\beta}=\frac{2 \sigma}{R},
$$

where $p_{\alpha}$ and $p_{\beta}$ are the bulk pressures of the liquid phase and the vapour phase, respectively, $\sigma$ is the surface tension of the liquid-gas interface and $R$ is the radius of the spherical droplet.

Equation (1) is now called the Young-Laplace-Kelvin equation or Laplace's equation. Laplace's theory of capillary phenomena is called molecular mechanics.

Since molecular mechanics failed to produce more quantitative results, this theory of gases and liquids did not develop further. Laplace and Young's works in the field of capillary phenomena were further developed by Poisson, Maxwell, van der Waals, Gibbs, etc. It was not until the establishment of thermodynamics that the capillary phenomena could be understood successfully.

The establishment of the second law of thermodynamics in the middle of the 19th century may be considered as a sign of the rise of thermodynamics. It is a natural idea to derive Laplace's equation (equation (1)) rigorously based on thermodynamics. Indeed, Gibbs developed a rigorous theory of the phenomena of surface tension by the methods of thermodynamics [10]. He derived and generalized Laplace's equation (equation (1)) based on the concept of a dividing surface.

In 1997, Mareschal et al [11] carried out molecular dynamics simulations to study the surface tensions of cylindrical droplets for the first time. Later, several research groups studied different properties of cylindrical droplets by molecular dynamics simulations [12-15].

However, there still exists controversy on the curvature dependence of surface tension of spherical and cylindrical droplets [12-18]. Further experimental, computational and theoretical investigations on the curvature dependence of surface tension of spherical and cylindrical droplets are needed.

The purpose of this paper is to derive a formula for the location of the surface of the tension and its surface tension for cylindrical droplets, which is somewhat different from those given by the previous authors, and can be carried out by methods of molecular dynamics simulations. As necessary preparation, in section 2, we will recall the definition of dividing surface in Gibbs' theory of capillary phenomena and the existing theories of surface tensions of cylindrical droplets.

The theoretical derivation is a typical application of Gibbs' theory of capillary phenomena. It is also a suitable problem in introductory lectures in the field of thermodynamic theory of capillary phenomena for graduate or postgraduate.

\section{Brief review of the definition of dividing surface and theories of surface tensions of cylindrical droplets}

\subsection{The definition of dividing surface}

First, we recall the definition of dividing surface in Gibbs' theory of capillary phenomena. Detailed discussion of the dividing surface can be found in [7, 10, 19].

Let us consider a liquid-vapour coexisting system with total volume $V$ and total molecular number $N$. Both experiments [20,21] and computer simulations [12, 13, 15, 22, 23] of liquidvapour systems have observed that it is in fact a continuous system with non-zero thickness liquid-vapour transition layers.

The gradient of density at any point $\vec{r}$ is nonzero in this liquid-vapor transition layers. According to Gibbs [10], we can always choose a parallel set of mathematical surfaces which 
are everywhere perpendicular to the density gradient. Any one of these mathematical surfaces can be chosen to be what Gibbs calls the dividing surface. An illustration of the dividing surface $S$ is shown in figure 2. This dividing surface $S$ divides this liquid-vapor coexisting system into two bulk phases, the liquid phase $\alpha$ and the vapour phase $\beta$.

Once the dividing surface $S$ is chosen, the original continuous system is represented by a model system: uniform liquid phase $\alpha$ with volume $V_{\alpha}$ and molecule number $N_{\alpha}$, uniform vapour phase $\beta$ with volume $V_{\beta}$ and molecule number $N_{\alpha}$ and geometrical dividing surface $S$ with molecule number $N_{s}$. We have

$$
V=V_{\alpha}+V_{\beta} .
$$

The extensive properties of phase $\alpha$ of the model system, that is, number of molecules $N_{\alpha}$, energy $U_{\alpha}$, etc, are defined by the equations

$$
N_{\alpha}=\rho_{\alpha} V_{\alpha}, \quad U_{\alpha}=\phi_{\alpha} V_{\alpha}, \quad \ldots,
$$

where $\rho_{\alpha}$ is the molecule number density of the phase $\alpha$ and $\phi_{\alpha}$ is the energy density of the phase $\alpha$.

In general, the sums $N_{\alpha}+N_{\beta}, U_{\alpha}+U_{\beta}$, etc are not equal to the total values of the whole system, $N, U$, etc. The differences are defined as the surface quantities

$$
N_{s}=N-N_{\alpha}-N_{\alpha}, \quad U_{s}=U-U_{\alpha}-U_{\alpha}, \quad \ldots,
$$

where $U_{s}$ is the energy of the surface.

The surface quantities $N_{s}$ and $U_{s}$ may be positive or negative. We can choose a dividing surface to make $N_{s}=0$, then, this one is called the equimolar surface.

Based on the concept of the dividing surface, Gibbs' generalized Laplace's equation for spherical droplets can be written as [7, 19]

$$
p_{\alpha}-p_{\beta}=\frac{2 \sigma(R)}{R}+\left[\frac{\mathrm{d} \sigma(R)}{\mathrm{d} R}\right] .
$$

where $R$ is the radius of a dividing surface, $\sigma(R)$ is the mechanical surface tension corresponding the dividing surface. The differential in square brackets denotes changes that follow from a notional change in the position of dividing surface [7, 19,24], which affects only the description of the system, and does not correspond to any physical change.

For the particular dividing surface such that the second term on the right-hand side of equation (5) vanishes, Gibbs called it a surface of tension [10]. From equation (5), we see that Laplace's equation (equation (1)) is valid only at the surface of tension. The first derivative vanishing means that the surface tension of the surface of tension is the extremum of the function of $R$.

In 1992, Nijmeijer et al [25] obtained the following generalized Laplace's equation for cylindrical droplets (refer to figures 1 and 2)

$$
p_{\alpha}-p_{\beta}=\frac{\sigma(R)}{R}+\left[\frac{\mathrm{d} \sigma(R)}{\mathrm{d} R}\right] .
$$

The same as for spherical droplets, the condition of the surface of the tension for cylindrical droplets is

$$
\left.\left[\frac{\mathrm{d} \sigma(R)}{\mathrm{d} R}\right]\right|_{R=R_{s}}=0
$$




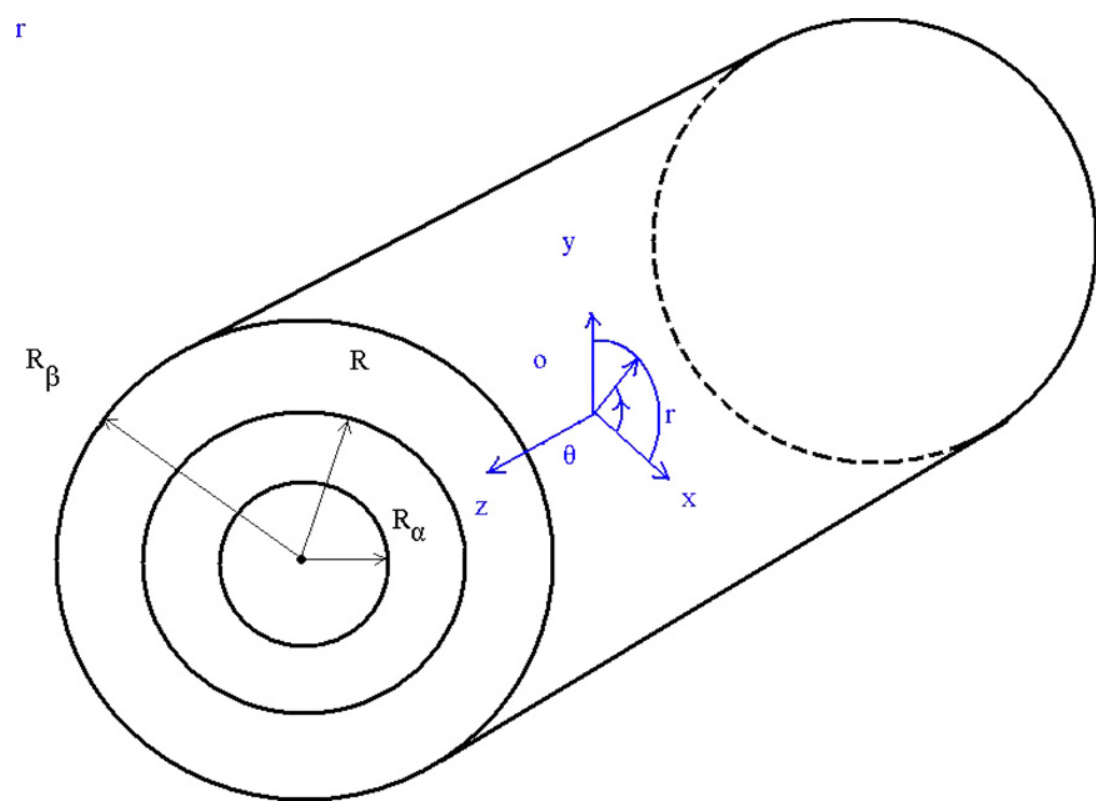

Figure 1. An illustration of a cylindrical droplet with its vapour.

vapor phase $\beta$

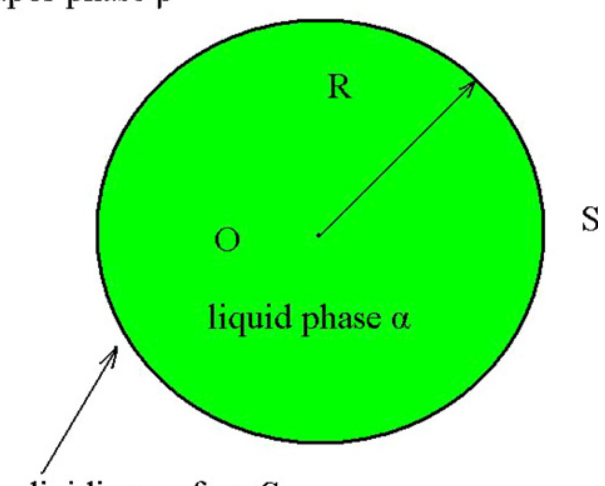

a dividing surface $\mathrm{S}$

Figure 2. An illustration of a dividing surface for a cylindrical droplet with its vapour.

\subsection{The existing theories of the surface tension of cylindrical droplets}

In this section, we recall some methods to calculate the surface tensions and radii of Gibbs' surface of tension of cylindrical droplets.

Consider a single-component cylindrical liquid droplet with its vapour. An illustration of this liquid-vapour system is shown in figure 1.

It is convenient to choose cylindrical coordinates for a cylindrical droplet system. We choose the centre of the cylindrical droplet as the origin of the cylindrical coordinates. Due to 
the cylindrical and transitional symmetry of the system, the physical quantities do not depend on $z$ and angle $\theta$. Thus, the pressure tensor can be written in the form

$$
P(r)=p_{i j}=\left(\begin{array}{ccc}
p_{N}(r) & 0 & 0 \\
0 & p_{T}(r) & 0 \\
0 & 0 & p_{T}(r)
\end{array}\right),
$$

where $r$ is the distance between the point and the axis of the cylindrical droplet, $p_{N}(r)$ and $p_{T}(r)$ are the normal and transverse components of the pressure tensor.

The pressure tensor $P(r)$ can also be written in the following form [15]:

$$
P(r)=p_{N}(r) \mathbf{e}_{r} \cdot \mathbf{e}_{r}+p_{T}(r) \mathbf{e}_{\theta} \cdot \mathbf{e}_{\theta}+p_{T}(r) \mathbf{e}_{z} \cdot \mathbf{e}_{z},
$$

where $\mathbf{e}_{r}, \mathbf{e}_{\theta}, \mathbf{e}_{z}$ are orthogonal unit vectors.

According to fluid mechanics [26-28], the condition of mechanical equilibrium is

$$
\nabla \cdot P=0,
$$

where $\nabla \cdot$ is the symbol of divergence operation of tensor.

From equation (10), we have [14]

$$
\frac{\partial p_{N}(r)}{\partial r}+\frac{p_{N}(r)-p_{T}(r)}{r}=0 \text {. }
$$

For convenience, we introduce the following notation:

$$
p_{\alpha \beta}(r, R)= \begin{cases}p_{\alpha}, & r<R, \\ p_{\beta}, & r>R,\end{cases}
$$

where $R$ is the radius of an arbitrary dividing surface, $r$ is the distance between the point and the axis of the cylindrical droplet.

Wei [14] and Kim et al [15] obtained the following result for the surface tension $\sigma(R)$ in the sense of the mechanical definition

$$
\sigma(R)=\int_{R_{\alpha}}^{R_{\beta}}\left(\frac{r}{R}\right)\left[p_{\alpha \beta}(r, R)-p_{T}(r)\right] \mathrm{d} r,
$$

where $R$ is the radius of an arbitrary dividing surface, $\sigma(R)$ is the mechanical surface tension with respect to an arbitrary dividing surface $R, R_{\alpha}<R$ and $R_{\beta}>R$ are two arbitrary positive integration boundaries.

In 2006, Kim et al [15] derived the following results for the surface tension and the radius of the surface of tension

$$
\begin{aligned}
& R_{s}^{2}=-\frac{1}{\left(p_{l}-p_{g}\right)} \int_{0}^{\infty} r^{2} \frac{\mathrm{d} p_{N}(r)}{\mathrm{d} R} \mathrm{~d} r \\
& \sigma^{2}\left(R_{s}\right)=-\left(p_{l}-p_{g}\right) \int_{0}^{\infty} r^{2} \frac{\mathrm{d} p_{N}(r)}{\mathrm{d} R} \mathrm{~d} r
\end{aligned}
$$

where $p_{N}(r)$ is the normal component of the pressure tensor, $R_{S}$ is the radius of the surface of tension, $\sigma\left(R_{S}\right)$ is the surface tension corresponding to the surface of tension $R_{S}$.

\section{A new method to calculate the radius of the surface of tension and the surface tension}

The purpose of this section is to give a new method to calculate the radius of the surface of tension $R_{S}$ and the surface tension $\sigma\left(R_{S}\right)$. The basis of this derivation is two basic concepts 
in Gibbs' thermodynamic theory of capillary phenomena, i.e., the dividing surface and the surface of tension.

Our starting point is a result about the mechanical surface tension $\sigma(R)$, i.e., equation (13). For convenience, we introduce the following notation:

$$
\begin{aligned}
& A=\int_{R_{\alpha}}^{R_{\beta}} r p_{T}(r) \mathrm{d} r, \\
& H(R)=\int_{R_{\alpha}}^{R_{\beta}} r p_{\alpha \beta}(r, R) \mathrm{d} r,
\end{aligned}
$$

where $R$ is the radius of an arbitrary dividing surface, $p_{\alpha}$ and $p_{\beta}$ are the pressures of the liquid phase and the vapour phase, respectively, $r$ is the distance between a point and the axis of the cylindrical droplet, $R_{\alpha}<R$ and $R_{\beta}>R$ are two arbitrary positive integration boundaries.

Then, using this notation and equations (16) and (17) in equation (13), we have

$$
\sigma(R)=\frac{H(R)-A}{R} .
$$

Now we first calculate the quantity $H(R)$ in equation (18). Direct integration of equation (17) gives

$$
\begin{aligned}
H(R) & =\int_{R_{\alpha}}^{R_{\beta}} r p_{\alpha \beta}(r, R) \mathrm{d} r \\
& =\int_{R_{\alpha}}^{R} r p_{\alpha} \mathrm{d} r+\int_{R}^{R_{\beta}} r p_{\beta} \mathrm{d} r \\
& =\frac{p_{\alpha}-p_{\beta}}{2} R^{2}-\frac{p_{\alpha}}{2} R_{\beta}^{2}+\frac{p_{\beta}}{2} R_{\beta}^{2} .
\end{aligned}
$$

From equations (18) and (19), we have

$$
\sigma(R)=\frac{p_{\alpha}-p_{\beta}}{2} R+\frac{p_{\beta} R_{\beta}^{2}-p_{\alpha} R_{\alpha}^{2}-2 A}{2 R} .
$$

From equation (20) we see that the mechanical surface tension $\sigma(R)$ exists for each dividing surface with radius $R$. Since there are infinitely many dividing surfaces, the value of the mechanical surface tension $\sigma(R)$ is not unique. For each dividing surface with radius $R$, the mechanical surface tension $\sigma(R)$ satisfies the generalized Laplace's equation (equation (6)).

Equation (20) can also be written as

$$
p_{\alpha}-p_{\beta}=\frac{2 \sigma(R)}{R}-\frac{p_{\beta} R_{\beta}^{2}-p_{\alpha} R_{\alpha}^{2}-2 A}{R^{2}} .
$$

Therefore, comparing with the generalized Laplace's equation (equation (6)), equation (21) may also be regarded as a generalized Laplace's equation.

Now we introduce the following notation:

$$
\begin{aligned}
& c=\frac{p_{\alpha}-p_{\beta}}{2}, \\
& a=\frac{p_{\beta} R_{\beta}^{2}-p_{\alpha} R_{\alpha}^{2}-2 A}{2} .
\end{aligned}
$$

Thus, using this notation and equations (16) and (17) in equation (20), we have

$$
\sigma(R)=c R+\frac{a}{R}
$$




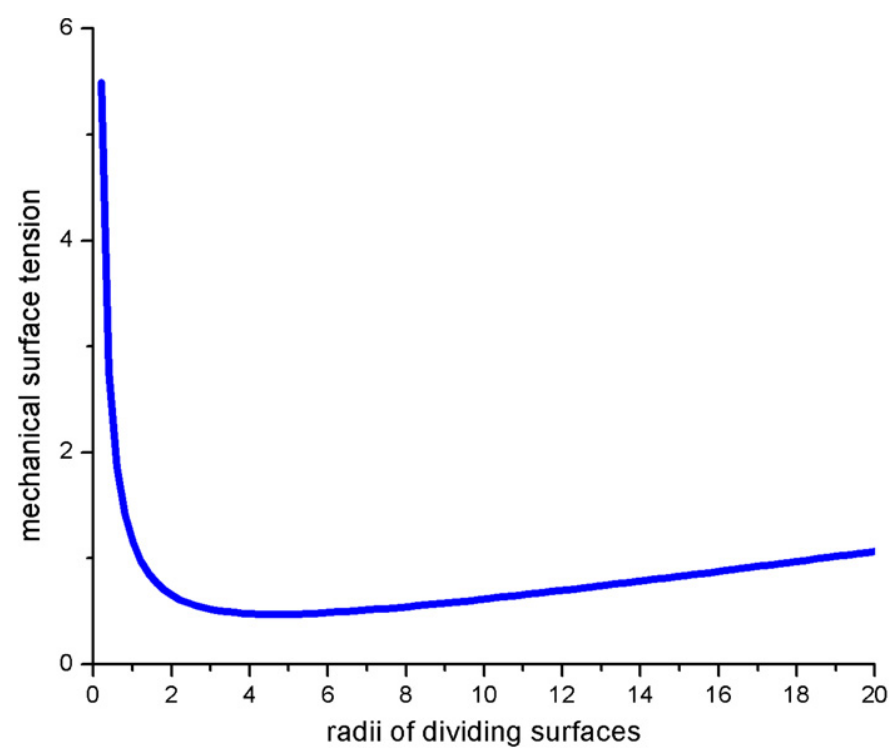

Figure 3. Mechanical surface tension described by equation (24). The data of the parameters in equation (24) are set to be $c=0.0505$ and $a=1.107$, which are taken from our molecular dynamics simulation results of the surface tensions of a cylindrical droplet system with 1105 argon molecules.

An illustration of the mechanical surface tension $\sigma(R)$ described in equation (24) is shown in figure 3. Figure 3 shows that the mechanical surface tension $\sigma(R)$ increases dramatically as the radius of dividing surface decreases. However, when the radius of dividing surface increases, the mechanical surface tension $\sigma(R)$ increases rather slowly. From figure 3, we see that there exists a minimum value of the mechanical surface tension $\sigma(R)$. This fact has already been demonstrated rigorously by Gibbs [10]. This minimum value of the mechanical surface tension $\sigma(R)$ is called surface tension and the dividing surface corresponding to surface tension is called the surface of tension by Gibbs [10].

Now let us determine the minimum value of the mechanical surface tension $\sigma(R)$ mathematically. From equations (7) and (24), we have

$$
R_{s}^{2}=\frac{a}{c}
$$

where $R_{S}$ is the radius of the surface of tension.

In general, we have $p_{\alpha}-p_{\beta} \geqslant 0$. Thus, if $a \geqslant 0$, then equation (25) becomes

$$
R_{s}=\sqrt{\frac{a}{c}}
$$

The value of $\sigma\left(R_{s}\right)$ is

$$
\sigma\left(R_{s}\right)=c R_{s}+\frac{a}{R_{s}} .
$$

From equations (26) and (27), we see that our method for surface tensions of cylindrical droplets can be carried out by molecular dynamics simulations.

To study the physical meaning of the two terms in equation (24), we obtain the following results from equations (26) and (27):

$$
a=\frac{R_{s} \sigma\left(R_{s}\right)}{2}, \quad c=\frac{\sigma\left(R_{s}\right)}{2 R_{s}} .
$$


From equation (28), equation (24) can be written as

$$
\sigma(R)=\frac{\sigma\left(R_{s}\right)}{2}\left[\frac{R_{s}}{R}+\frac{R}{R_{s}}\right] .
$$

The physical origin of the mechanical surface tension $\sigma(R)$ shown in figure 3 is illustrated by equation (29).

\section{Conclusion}

The history of Laplace's equations for spherical and cylindrical droplets and the concept of dividing surface in Gibbs' thermodynamic theory of capillary phenomena are briefly reviewed. The existing theories of surface tension of cylindrical droplets are briefly reviewed too. For cylindrical droplets, a new method to calculate the radius of the surface of tension and the surface tension is given. This method is suitable to be carried out by molecular dynamics simulations.

The theoretical derivation of the location of the surface of tension of a cylindrical droplet is a typical and suitable problem in introductory lectures in the field of thermodynamic theory of capillary phenomena. It may be helpful for graduate or postgraduate beginners to grasp Gibbs' concepts of dividing surface and surface of tension. Nowadays, the capillary phenomena in nanoscale are a field full of puzzles, controversies and challenges. Since so many great scientists in history connected with capillary phenomena, the historical materials presented here may be valuable to encouraging graduate or postgraduate students to take part in the investigations of capillary phenomena in nanoscale.

\section{Acknowledgments}

This work was supported by the National Natural Science Foundation of China (grant nos. 10472128 and 10772189) and the Knowledge Innovation Program of Chinese Academy of Sciences.

\section{References}

[1] Pomeau Y and Villermaux E 2006 Phys. Today 5939

[2] Newton I 1952 Opticks (New York: Dover)

[3] Rowlinson J S 1992 Fundamentals of Inhomogeneous Fluids ed D Henderson (New York: Dekker) pp 1-22

[4] Rutherford E 1965 The Collected Papers of Lord Rutherford of Nelson vol 2 (London: Allen and Unwin)

[5] Yan K-N 1993 Atomic Theory and Mordern Science (Beijing: Higher Education Press)

[6] Laplace P-S 1805 Traîté de mécanique céleste, Supplement to vol 4 (Paris: Courcier)

[7] Rowlinson J S and Widom B 1982 Molecular Theory of Capillary (Oxford: Clarendon)

[8] Thomson W 1858 Phil. Trans. R. Soc. Lond. 9255

[9] Thomson W 1859 Phil. Mag. 1761

[10] Gibbs J W 1961 The Scientific Papers of J W Gibbs vol 1 (New York: Dover)

[11] Mareschal M, Baus M and Lovett R 1997 J. Chem. Phys. 106645

[12] El Bardouni H et al 2000 J. Chem. Phys. 1139804

[13] van Giessen A E and Blokhuis E M 2002 J. Chem. Phys. 116302

[14] Wei J-A 2005 Theories and molecular dynamics simulations of cylindrical droplets and their contact phenomena on solid surfaces Master Thesis

[15] Kim B G, Lee J S, Han M and Park S 2006 Nanoscale Microscale Thermophys. Eng. 10283

[16] Baidakov V G and Boltachev G Sh 2004 J. Chem. Phys. 1218594

[17] Lei Y A, Bykov T, Yooo S and Zeng X C 2005 J. Am. Chem. Soc. 12715346

[18] Blokhuis E M and Kuipers J 2006 J. Chem. Phys. 124074701

[19] Ono S and Kondo S 1960 Encyclopedia of Physics ed S Flugge vol 10 (Berlin: Springer)

[20] Checco A, Guenoun P and Daillant J 2003 Phys. Rev. Lett. 91186101

[21] Checco A, Gang O and Ocko M 2006 Phys. Rev. Lett. 96056104 
[22] Chapela G A et al 1977 J. Chem. Soc. Faraday Trans. II 731133

[23] Thompson S M, Gubbins K E, Walton J P R B, Chantry R A R and Rowlinson J S 1984 J. Chem. Phys. 81530

24] Kondo S 1956 J. Chem. Phys. 25662

[25] Nijmeijer M J P, Bruin C, van Woerkom A B and Bakker A F 1992 J. Chem. Phys. 96565

[26] Yih C-S 1969 Fliud Mechanics (New York: McGraw-Hill)

[27] Currie I G 2003 Fundamental Mechanics of Fluids (Cambridge: Cambridge University Press)

[28] Wu W-Y 1982 Fluid Mechanics vol 1 (Beijing: Beijing University Press) 